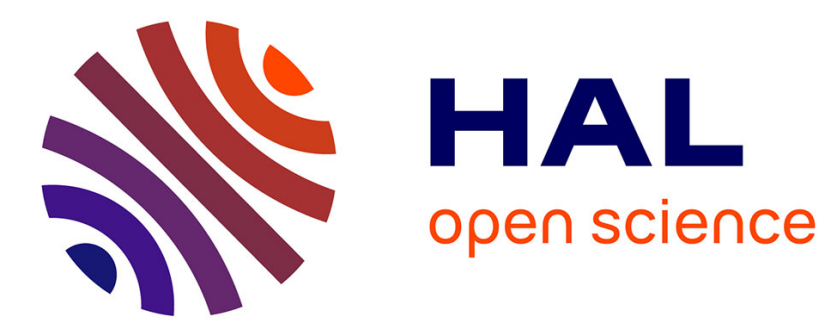

\title{
Robustness Analysis with Respect to Exogenous Perturbations for Flatness-Based Exact Feedforward Linearization
}

Hagenmeyer Veit, Delaleau Emmanuel

\section{To cite this version:}

Hagenmeyer Veit, Delaleau Emmanuel. Robustness Analysis with Respect to Exogenous Perturbations for Flatness-Based Exact Feedforward Linearization. 2009. hal-00431712

\section{HAL Id: hal-00431712 \\ https://hal.science/hal-00431712}

Preprint submitted on 12 Nov 2009

HAL is a multi-disciplinary open access archive for the deposit and dissemination of scientific research documents, whether they are published or not. The documents may come from teaching and research institutions in France or abroad, or from public or private research centers.
L'archive ouverte pluridisciplinaire HAL, est destinée au dépôt et à la diffusion de documents scientifiques de niveau recherche, publiés ou non, émanant des établissements d'enseignement et de recherche français ou étrangers, des laboratoires publics ou privés. 


\title{
Robustness Analysis with Respect to Exogenous Perturbations for Flatness-Based Exact Feedforward Linearization
}

\author{
Veit Hagenmeyer, Emmanuel Delaleau
}

\begin{abstract}
A methodology to analyze robustness with respect to exogenous perturbations for exact feedforward linearization based on differential flatness is presented. The analysis takes into consideration the tracking error equation and makes thereafter use of a stability result by Kelemen coupled with results issued from interval analysis. This turns exact feedforward linearization based on differential flatness into a general control methodology for flat systems.
\end{abstract}

\section{Index Terms}

Nonlinear systems, tracking control, differential flatness, exact feedforward linearization, stability analysis, exogenous perturbations, robustness.

\section{INTRODUCTION}

$\mathbf{I}$ $\mathrm{N}$ order to emphasize that differential flatness ${ }^{1}$ can also be considered from a trajectory point of view (and not only from a feedback linearization one), the authors have presented the concept of exact feedforward linearization based on differential flatness in [3]. This concept allows to design the control of nonlinear flat systems as a specific combination of a nominal feedforward input and a simple feedback stabilizing control. In [4], the authors presented furthermore a method to analyze parametric robustness of the proposed control scheme. Thus the results of [3], [4] and those presented here turn exact feedforward linearization based on differential flatness into a general control methodology for flat systems.

The purpose of the present contribution is to introduce a robustness analysis methodology with respect to exogenous perturbations and time-varying parameters. It is presented by its application to exact feedforward linearization in the case of the use of an extended PID-like control for the feedback part. A direct analysis of the tracking error equation, to which the stability result by Kelemen [5] is applied, leads to the main result of this contribution.

The contribution is organized as follows: Sec. II generalizes the notion of exact feedforward linearization based on differential flatness to the case of exogenous perturbations and time-varying parameters. Using this result, Sec. III establishes a specific control law design making use of the nominal values of the exogenous perturbations and time-varying parameters. This method leads to a specific tracking error equation when exogenous perturbations are considered; it is discussed in Sec. IV. In Sec. V robustness is proven for the presented control strategy.

\section{Exact Feedforward Linearization in the ConteXt of EXogenous Perturbations}

Differential flatness is a structural property of a class of nonlinear systems, for which, roughly speaking, all system variables can be written in terms of a set of specific variables (the so-called flat outputs) and their derivatives. In [3] it is demonstrated, that these systems are linearizable by a nominal feedforward

V. Hagenmeyer is with the BASF SE, WL - C100, 67056 Ludwigshafen, Germany. e-mail: veit.hagenmeyer@basf.com.

E. Delaleau is with the EA 4325 Laboratoire brestois de mécanique et des systèmes, École nationale d'ingénieurs de Brest, Technopôle Brest-Iroise, CS 73862, 29238 Brest Cedex 3, France. Université européenne de Bretagne, Brest. e-mail: delaleau@enib.fr.

${ }^{1}$ See [1], [2] for an overview on differentially flat control systems. 
if the initial condition is known. Exact feedforward linearization is recalled in the context of exogenous perturbations and time varying parameters after the following brief representation of differential flatness:

For the sake of simplicity, only SISO flat systems are considered in the sequel without loss of generality (cf. [3] for the MIMO case). Given the SISO nonlinear system

$$
\dot{\mathbf{x}}(t)=\mathbf{f}(\varpi(t), \mathbf{x}(t), u(t))
$$

with $\mathbf{x}(0)=\mathbf{x}_{0}$. Thereby it holds that the time $t \in \mathbb{R}$, the state $\mathbf{x}(t) \in \mathbb{R}^{n}$, and the input $u(t) \in \mathbb{R}$. The exogenous perturbations (or time-varying system parameters) $t \mapsto \varpi(t) \in \mathbb{R}^{q}$ are supposed to be modeled either by known sufficiently smooth time functions in the time-varying parameter case or close to physical reality by consecutive Heaviside jumps with microstructure in the exogenous perturbation case. Heaviside jumps with microstructure are a so-called class of Heaviside generalized functions [6]. Roughly speaking, Heaviside jumps with microstructure have an infinitely smooth jump transition at the origin (and in an $\epsilon$-neighborhood around it), therefore $\varpi \in \mathcal{C}^{\infty}$. The exogenous perturbations or time-varying parameters are supposed to be partitionable as:

$$
\varpi^{(j)}(t)=\varpi_{o}^{(j)}(t)+\tilde{\varpi}^{(j)}(t), \quad \tilde{\varpi}_{i}^{(j)}(t) \in\left[\underline{\varpi}_{i}^{(j)}, \bar{\varpi}_{i}^{(j)}\right], \quad i=1, \ldots, q, \quad j=0, \ldots, n-1
$$

where $\varpi_{o}$ is the nominal value of the exogenous perturbations ${ }^{2}$ or time-varying parameters and their time derivatives respectively. In (1) the vector field $\mathbf{f}: \mathbb{R}^{q} \times \mathbb{R}^{n} \times \mathbb{R} \rightarrow \mathbb{R}^{n}$ is considered to be smooth. The system (1) is said to be (differentially) flat ${ }^{3}$ iff for all $\varpi(t)$ satisfying (2) there exists a flat output $z \in \mathbb{R}$, such that

$$
\begin{aligned}
z & =F(\mathbf{x}) \\
\mathbf{x} & =\phi\left(\boldsymbol{\Pi}, z, \dot{z}, \ldots, z^{(n-1)}\right) \\
u & =\psi\left(\boldsymbol{\Pi}, z, \dot{z}, \ldots, z^{(n)}\right)
\end{aligned}
$$

with

$$
\begin{aligned}
\Pi & =\left[\Pi_{1}, \Pi_{2}, \ldots, \Pi_{q}\right]^{T} \\
& =\left[\varpi_{1}, \dot{\varpi}_{1}, \ddot{\varpi}_{1}, \ldots, \varpi_{1}^{(n-1)}, \varpi_{2}, \dot{\varpi}_{2}, \ldots, \varpi_{q}, \ldots, \varpi_{q}^{(n-1)}\right]^{T}
\end{aligned}
$$

(Correspondingly, $\boldsymbol{\Pi}_{o}$ denotes the nominal value of $\Pi$.) Thereby $F, \phi$ and $\psi$ are smooth functions at least in open subsets ${ }^{4}$ of $\mathbb{R}^{n}, \mathbb{R}^{n(q+1)}$ or $\mathbb{R}^{n(q+1)+1}$, respectively. For the sake of simplicity, it is furthermore assumed in the sequel that the flat output is independent of $\Pi$ in two ways: in (3), first the function $F$ is independent of $\Pi$, and second $F$ is the same function for all $\Pi$ defined by (2). ${ }^{5}$

The equations ${ }^{6}$ (4) and (5) yield - under the assumption of the knowledge of $\Pi$ - that for every given trajectory of the flat output $t \mapsto z(t)$, the evolution of all other variables of the system $t \mapsto \mathbf{x}(t)$ and $t \mapsto u(t)$ are given without integration of any differential equation. Moreover, for a sufficiently smooth desired trajectory of the flat output $t \mapsto z^{*}(t)$, eqn. (5) can be used to design the corresponding feedforward $u^{*}(t)$ directly for the nominal $\Pi_{o}$. The trajectory $z^{*}$ is called the nominal trajectory, while the trajectory $u^{*}$ is called the nominal control. The family of nominal feedforwards is given by

$$
u^{*}(t)=\psi\left(\boldsymbol{\Pi}_{o}(t), z^{*}(t), z^{*}(t), \ldots, z^{*(n)}(t)\right)
$$

\footnotetext{
${ }^{2}$ If there is no a priori information given about the nominal time profile of the exogenous perturbation, let $\varpi_{o i}(t)=\varpi_{o i}^{*}, i=1, \ldots, q$, where the $\varpi_{o i}^{*}$ are the constant expected mean values of the respective perturbations.

${ }^{3}$ This definition is an adaptation from [7], [8].

${ }^{4}$ The eventual singularities appearing in $F, \phi$ and $\psi$ are outside of this open subsets.

${ }^{5}$ This assumption is not very restrictive in the case of real systems. The flat output can often be chosen to be a physical variable (a position, a current, a voltage, a temperature, etc.), which is independent of any unknown parameter. The range of the uncertainty of the parameter set defined by (2) does not change generically the dynamic structure of the system. Moreover, the whole robustness analysis in the sequel can also be led without this assumption, but then (31) becomes a Liouvillian system and the notationally nice structure of (35) is lost.

${ }^{6}$ The maximal number of derivatives of $z$ in (4) and (5) respectively are due to the results of [9], [10] (see more details below in the proof of Theorem 2.1).
} 
that is, for each admissible nominal trajectory $z^{*}$, there is a nominal feedforward $u^{*}$.

Definition 2.1: The initial condition of the desired trajectory of the flat output $t \mapsto z^{*}(t)$ is defined by $\mathbf{z}_{0}^{*}=\left[z^{*}(0), \dot{z}^{*}(0), \ldots, z^{*(n-1)}(0)\right]^{T}$. It is consistent with the initial condition $\mathbf{x}_{0}$, if $\mathbf{x}_{0}=\phi\left(\boldsymbol{\Pi}_{o}(0), \mathbf{z}_{0}^{*}\right)$ (c.f. eqn. (4)).

Exact feedforward linearization based on differential flatness established in [3] can be generalized to the case of exogenous perturbations as follows: to this end, interpret (1) and (3) as a control system with input $u$, state $\mathbf{x}$ and output $z$.

Theorem 2.1: If the desired trajectory of the flat output is consistent with the initial condition $\mathrm{x}_{0}$, and $\Pi=\Pi_{o}$ holds for all times, the application of the nominal control (6) to the control system (1) yields an output that satisfies $z(t)=z^{*}(t), \forall t \geq 0$, i.e. the output of the nonlinear system (1)-(3) corresponds to the output of a Brunovský system driven by $z^{*(n)}$ and initialized at $\mathbf{z}_{0}^{*}$.

Proof: Considering the results of [9], [10], it is easy to show, that every $\operatorname{SISO}^{7}$ flat system can be represented as follows. Setting

$$
\mathbf{z}=\left[z, \dot{z}, \ldots, z^{(n-1)}\right]^{T}=\left[z_{1}, z_{2}, \ldots, z_{n}\right]^{T}
$$

the system (1) can be transformed via the well defined state transformation

$$
\mathbf{z}=\boldsymbol{F}\left(\boldsymbol{\Pi}_{o}, \mathbf{x}\right)
$$

(where $\boldsymbol{F}$ is equal to the solution of (4)) w.r.t. z, into the control normal form

$$
\begin{aligned}
\dot{z}_{i}(t) & =z_{i+1}(t), \quad i \in\{1, \ldots, n-1\} \\
\dot{z}_{n}(t) & =\alpha\left(\boldsymbol{\Pi}_{o}(t), \mathbf{z}(t), u(t)\right)
\end{aligned}
$$

where $\alpha$ is also smooth with respect to its arguments. (Recall that we assume here that $\Pi=\Pi_{o}$.) The initial condition of (9) corresponds to the one of (1) as $\mathbf{z}_{0}=\mathbf{z}(0)=\boldsymbol{F}\left(\boldsymbol{\Pi}(0), \mathbf{x}_{0}\right)$ from (8). Remark, that a sort of matching condition [11] is always satisfied in the sense, that the input, the perturbation and its derivatives enter the system equation at the same spot. Applying the feedforward (6) to the differentially flat system given by (1) is equivalent to the application of $u^{*}(t)$ of (6) to (9), which results in

$$
\begin{aligned}
\dot{z}_{i}(t) & =z_{i+1}(t), \quad i \in\{1, \ldots, n-1\} \\
\dot{z}_{n}(t) & =\alpha\left(\boldsymbol{\Pi}_{o}(t), \mathbf{z}(t), \psi\left(\boldsymbol{\Pi}_{o}(t), \mathbf{z}^{*}(t), \dot{z}_{n}^{*}(t)\right)\right)
\end{aligned}
$$

Thus (10) and (1)-(3)-(6) have the same solution $t \mapsto \mathbf{z}(t)$.

The proof proceeds by establishing that (10) admits the same solution as the Brunoský form:

$$
\begin{aligned}
\dot{z}_{i}(t) & =z_{i+1}(t), \quad i \in\{1, \ldots, n-1\} \\
\dot{z}_{n}(t) & =v(t)
\end{aligned}
$$

when $v(t)=z^{*(n)}(t)$ and $\mathbf{z}(0)=\mathbf{z}_{o}^{*}$.

By iterative integrations, one sees that the application of $v(t)=z^{*(n)}$ from the initial condition $\mathbf{z}_{0}^{*}$ to (11) implies that $\mathbf{z}(t)=\mathbf{z}^{*}(t)$.

Write now the differential equation satisfied by $\epsilon=\mathbf{z}-\mathbf{z}^{*}$, where $\mathbf{z}$ is any solution of (10) and $\mathbf{z}^{*}$ is the solution of (11) when $\mathbf{z}(0)=\mathbf{z}_{0}^{*}$ and $v(t)=z^{*}(t)$. We obtain:

$$
\begin{aligned}
\dot{\epsilon}_{i}(t) & =\epsilon_{i+1}(t), \quad i \in\{1, \ldots, n-1\} \\
\dot{\epsilon}_{n}(t) & =\alpha\left(\boldsymbol{\Pi}_{o}(t), \mathbf{z}^{*}(t)+\epsilon(t), \psi\left(\boldsymbol{\Pi}_{o}(t), \mathbf{z}^{*}(t), \dot{z}_{n}^{*}(t)\right)\right)-\dot{z}_{n}^{*}(t)
\end{aligned}
$$

In view of (9) it is evident, that (5) is the solution for $u$ of

$$
0=\alpha\left(\boldsymbol{\Pi}_{o}, \mathbf{z}, u\right)-\dot{z}_{n}
$$

\footnotetext{
${ }^{7}$ The proof is written in the SISO case for notational convenience. A similar proof for the MIMO case can be led considering [3].
} 
with $\dot{z}_{n}=z^{(n)}$. Hence one gets

$$
\alpha\left(\boldsymbol{\Pi}_{o}, \mathbf{z}, \psi\left(\boldsymbol{\Pi}, \mathbf{z}, \dot{z}_{n}\right)\right)=\dot{z}_{n}
$$

Consequently, $\epsilon=0$ is an equilibrium point of (12) and thus the solution of (10) initialized at $\mathbf{z}_{o}^{*}$ is also $\mathbf{z}^{*}$.

\section{EXACT FEEDFORWARD LINEARIZATION AND CONTROL LAW DESIGN}

In [3], exact feedforward linearization is used to design a specific PID-like stabilization of the desired trajectory. The control law consists of two parts, a feedforward part (6), and a PID-like feedback part that takes the tracking error into account. Since in [3] the control law is implicitly designed for the case of nominal parameters and nominal exogenous perturbations, the assumption throughout this section is that the exogenous perturbations coincide with the nominal ones, that is $\Pi=\Pi_{o}$. The structure of the combination of both parts can then be represented as in [3]:

Since $v=\dot{z}_{n}^{*}$ is the input of the Brunovský form in Theorem 2.1, the new input $v$ is designed as

$$
v=\dot{z}_{n}^{*}+\Lambda(\overline{\mathbf{e}})
$$

where the tracking error $\mathbf{e}=\left[e_{1}, e_{2}, \ldots, e_{n}\right]^{T}$ and the augmented tracking error $\overline{\mathbf{e}}=\left[e_{0}, e_{1}, e_{2}, \ldots, e_{n}\right]^{T}$ are defined by (remember $\mathbf{z}=\boldsymbol{F}\left(\boldsymbol{\Pi}_{o}, \mathbf{x}\right)$ of (8))

$$
e_{i}=z_{i}-z_{i}^{*}, \quad i \in\{1, \ldots, n\} ; \quad e_{0}=\int_{0}^{t} e_{1}(\tau) d \tau
$$

The extended PID-like ${ }^{8}$ feedback part is

$$
\Lambda(\overline{\mathbf{e}})=\lambda_{0} \int_{0}^{t} e_{1}(\tau) d \tau+\sum_{i=1}^{k+1} \lambda_{i} e_{i}(t)=\sum_{i=0}^{k+1} \lambda_{i} e_{i}(t)
$$

where $k$ is a fixed integer in $\{0, \ldots, n-1\}$. Thus, the whole control structure can be denoted by

$$
u=\psi\left(\boldsymbol{\Pi}_{o}, \mathbf{z}^{*}, v\right)=\psi\left(\boldsymbol{\Pi}_{o}, \mathbf{z}^{*}, \dot{z}_{n}^{*}+\sum_{i=0}^{k+1} \lambda_{i} e_{i}\right)
$$

This structure consists of a specific combination of a nonlinear feedforward part based on differential flatness, and a simple linear feedback part of extended PID type. Remark that this control structure represents a truly nonlinear control.

The advantage of the structure (18) becomes evident in view of (14). On the desired trajectory ${ }^{9}$, one gets $\alpha\left(\boldsymbol{\Pi}_{o}, \mathbf{z}^{*}, \psi\left(\boldsymbol{\Pi}_{o}, \mathbf{z}^{*}, v\right)\right)=v$ and therefore

$$
\frac{\partial \alpha\left(\boldsymbol{\Pi}_{o}, \mathbf{z}^{*}, \psi\left(\boldsymbol{\Pi}_{o}, \mathbf{z}^{*}, v\right)\right)}{\partial v}=1
$$

This property shows its effect on the structure of the error equation, which results from the application of the control law (18) to (9):

$$
\begin{aligned}
\dot{z}_{i} & =z_{i+1}, \quad i \in\{1, \ldots, n-1\} \\
\dot{z}_{n} & =\alpha\left(\boldsymbol{\Pi}_{o}, \mathbf{z}, \psi\left(\boldsymbol{\Pi}_{o}, \mathbf{z}^{*}, \dot{z}_{n}^{*}+\sum_{i=0}^{k+1} \lambda_{i} e_{i}\right)\right)
\end{aligned}
$$

\footnotetext{
8"'Extended" in the sense that multiple derivatives of the error can be used, where the D-parts are in the higher order errors $e_{i}, k \geq 1$, cf. (16) and (7). Thereby it is important to remark that these derivatives are not obtained by successive derivation of a (possibly) noisy signal, but they are calculated using the measured state (or the necessary part of it), eqn. (8) with $\Pi=\Pi_{o}$, and eqn. (16).

${ }^{9}$ Being on the desired trajectory means $\mathbf{z}=\mathbf{z}^{*}$, that is also $z=z^{*}$ and correspondingly $\mathbf{x}=\mathbf{x}^{*}(c f$. (4)).
} 
Using (20) and (16), the corresponding tracking error system can be denoted as

$$
\begin{aligned}
& \dot{e}_{i}=e_{i+1}, \quad i \in\{0, \ldots, n-1\} \\
& \dot{e}_{n}=\alpha\left(\boldsymbol{\Pi}_{o}, \mathbf{e}+\mathbf{z}^{*}, \psi(\boldsymbol{\Pi}_{o}, \mathbf{z}^{*}, \underbrace{\dot{z}_{n}^{*}+\sum_{i=0}^{k+1} \lambda_{i} e_{i}}_{v})-\dot{z}_{n}^{*}\right.
\end{aligned}
$$

The linearized system around the desired trajectory $(\overline{\mathbf{e}}=\mathbf{0})$ is then given by

$$
\dot{\overline{\mathbf{e}}}_{\delta}=\left(\left[\begin{array}{ccccc}
0 & 1 & 0 & \cdots & 0 \\
0 & 0 & 1 & \cdots & 0 \\
\vdots & & & \ddots & \vdots \\
0 & 0 & 0 & \cdots & 1 \\
\gamma_{0} & \gamma_{1} & \gamma_{2} & \cdots & \gamma_{n}
\end{array}\right]+\left[\begin{array}{c}
0 \\
0 \\
\vdots \\
0 \\
1
\end{array}\right]\left[\begin{array}{llll}
\nu_{0} & \nu_{1} & \cdots & \nu_{n}
\end{array}\right]\right) \overline{\mathbf{e}}_{\delta}
$$

where (remember the arguments of $\alpha\left(\boldsymbol{\Pi}_{o}, \mathbf{z}, u\right)$ in (9))

$$
\gamma_{i}=\left.\frac{\partial \alpha}{\partial z_{i}} \frac{\partial z_{i}}{\partial e_{i}}\right|_{\overline{\mathbf{e}=0}}=\left.\frac{\partial \alpha}{\partial z_{i}}\right|_{\mathbf{z}=\mathbf{z}^{*}}
$$

since $\frac{\partial z_{i}}{\partial e_{i}}=1$ in view of (16), and furthermore (defining $\lambda_{i}=0, i \in\{k+2, \ldots, n\}$ if necessary)

$$
\nu_{i}=\left.\frac{\partial \alpha}{\partial u} \frac{\partial u}{\partial v} \frac{\partial v}{\partial e_{i}}\right|_{\overline{\mathbf{e}}=\mathbf{0}}=\left.\frac{\partial v}{\partial e_{i}}\right|_{\overline{\mathbf{e}}=\mathbf{0}}=\lambda_{i}
$$

since $\left.\frac{\partial \alpha}{\partial u} \frac{\partial u}{\partial v}\right|_{\overline{\mathbf{e}}=\mathbf{0}}=\left.\frac{\partial \alpha}{\partial v}\right|_{\mathbf{\mathbf { e }}=\mathbf{0}}=1$ in view of (19) and (21).

When using full state information, that is $k=n-1$ in (17), the structure of (22) shows that all coefficients of the characteristic polynomial of the linearized system around the desired trajectory can be modified, since it can be written as

$$
\begin{aligned}
& s^{n+1}-\gamma_{n} s^{n}-\cdots-\gamma_{0}= \\
& s^{n+1}-\left(\left.\frac{\partial \alpha}{\partial z_{n}}\right|_{\mathbf{z}=\mathbf{z}^{*}}+\lambda_{n}\right) s^{n}-\left(\left.\frac{\partial \alpha}{\partial z_{n-1}}\right|_{\mathbf{z}=\mathbf{z}^{*}}+\lambda_{n-1}\right) s^{n-1}-\cdots-\lambda_{0}
\end{aligned}
$$

Remark 3.1: When considering time-dependent controller coefficients $\lambda_{i}=\lambda_{i}\left(\mathbf{z}^{*}(t), \dot{z}_{n}^{*}(t)\right), i \in\{0, \ldots, n\}$, it is evident that the poles of this system can be placed for a given characteristic polynomial. This could be interpreted as a dynamical kind of pseudo-linearization [12] around the desired trajectory or a desired flatness based gain scheduling.

In [3], several structural properties of the application of (18) to (1) are discussed in detail implicitly for nominal constant parameters $\Pi(t)=\Pi_{o}$. In this case, one of the main results of [3] consists in a stability proof for (1) under (18). For nominal time-varying parameters $\Pi(t)=\Pi_{o}(t)$ the stability proof of [3] can easily be extended by including the nominal $\Pi_{o}(t)$ into the input vector of the error equation. ${ }^{10}$ Thus in the following, it is assumed, that (18) guarantees stability of (1) for $\Pi(t)=\Pi_{o}(t)$.

\footnotetext{
${ }^{10}$ In the same manner as it is also done for the main result of this contribution, see Theorem 5.1 below.
} 


\section{The ERror EquATION OF EXACT FEEDFORWARd LINEARIZATION UNDER EXOGENOUS PERTURBATIONS}

In the case $\Pi \neq \Pi_{o}$ as generally defined in (2), it still makes sense to apply the controller of (18) if the difference between the nominal exogenous perturbations $\Pi_{o}$ and the real ones is "not too big". Thereby a difficulty appears for the feedback part of (17), since the exact value of $\mathbf{z}$ cannot be reconstructed by the knowledge of $\mathbf{x}$ (remember $\mathbf{z}=\boldsymbol{F}(\boldsymbol{\Pi}, \mathbf{x})$ of (8)). Therefore, the following approximate state ${ }^{11}$ has to be considered

$$
\zeta=\boldsymbol{F}\left(\boldsymbol{\Pi}_{o}, \mathbf{x}\right)
$$

Thus the tracking error used for the feedback part (17) is defined as

$$
\tilde{e}_{i}=\zeta_{i}-z_{i}^{*}, \quad i \in\{1, \ldots, n\}, \quad \tilde{e}_{0}=\int_{0}^{t} \tilde{e}_{1}(\tau) d \tau
$$

Notice that for $j=0,1, \tilde{e}_{j}=e_{j}$, since the flat output $z$ is independent of $\varpi$, see (3). Using (27), the nonlinear control law (18) has to be rewritten as

$$
u=\psi\left(\boldsymbol{\Pi}_{o}, \mathbf{z}^{*}, v\right)=\psi\left(\boldsymbol{\Pi}_{o}, \mathbf{z}^{*}, \dot{z}_{n}^{*}+\sum_{i=0}^{k+1} \lambda_{i} \tilde{e}_{i}(t)\right)
$$

To study the robustness of the system (1) under the control law (28) in the vicinity of the desired trajectory, the control law (28) is substituted into (9), which yields

$$
\begin{aligned}
\dot{z}_{i} & =z_{i+1}, \quad i \in\{1, \ldots, n-1\} \\
\dot{z}_{n} & =\alpha\left(\boldsymbol{\Pi}, \mathbf{z}, \psi\left(\boldsymbol{\Pi}_{o}, \mathbf{z}^{*}, \dot{z}_{n}^{*}+\sum_{i=0}^{k+1} \lambda_{i} \tilde{e}_{i}(t)\right)\right) \\
& =\alpha\left(\boldsymbol{\Pi}, \mathbf{z}, \psi\left(\boldsymbol{\Pi}_{o}, \mathbf{z}^{*}, \dot{z}_{n}^{*}+\Lambda(\tilde{\overline{\mathbf{e}}})\right)\right)
\end{aligned}
$$

where $\tilde{\overline{\mathbf{e}}}=\left[\tilde{e}_{0}, \tilde{e}_{1}, \tilde{e}_{2}, \ldots, \tilde{e}_{n}\right]^{T}$. To find the real tracking error equation in $\mathbf{e}=\mathbf{z}-\mathbf{z}^{*}$, the following relation between $\tilde{\mathbf{e}}=\left[\tilde{e}_{1}, \tilde{e}_{2}, \ldots, \tilde{e}_{n}\right]^{T}$ and e can be established considering (8) and (4)

$$
\begin{aligned}
\tilde{\mathbf{e}} & =\boldsymbol{F}\left(\boldsymbol{\Pi}_{o}, \mathbf{x}\right)-\mathbf{z}^{*}=\boldsymbol{F}\left(\boldsymbol{\Pi}_{o}, \boldsymbol{\phi}(\boldsymbol{\Pi}, \mathbf{z})\right)-\mathbf{z}^{*} \\
& =\boldsymbol{F}\left(\boldsymbol{\Pi}_{o}, \boldsymbol{\phi}\left(\boldsymbol{\Pi}, \mathbf{e}+\mathbf{z}^{*}\right)\right)-\mathbf{z}^{*}
\end{aligned}
$$

Since $\tilde{e}_{0}=e_{0}$, the feedback part $\Lambda(\tilde{\overline{\mathbf{e}}})$ can thus be written as $\tilde{\Lambda}(\overline{\mathbf{e}})$. Then, using (29) the augmented tracking error system in $\overline{\mathbf{e}}$ can be denoted as

$$
\begin{aligned}
\dot{e}_{i} & =e_{i+1}, \quad i \in\{0, \ldots, n-1\} \\
\dot{e}_{n} & =\alpha\left(\boldsymbol{\Pi}, \mathbf{e}+\mathbf{z}^{*}, \psi\left(\boldsymbol{\Pi}_{o}, \mathbf{z}^{*}, \dot{z}_{n}^{*}+\tilde{\Lambda}(\overline{\mathbf{e}})\right)-\dot{z}_{n}^{*}\right.
\end{aligned}
$$

An analysis of robust stability of (31) will be carried out in the following section.

\section{Robustness AnAlysis}

In this section it is shown, through the study of the error equation (31), how stability of the control strategy (28) applied to the system (1) can be analyzed. For the sake of generality, full state information is used in the PID-part, that is $k=n-1$ in (28) for the sequel. How this assumption can be relaxed thereafter is described at the end of this section.

The augmented tracking error equation (31) can be written structurally as (defining $\underline{\mathbf{z}}^{*}=\left[z_{1}^{*}, \ldots, z_{n}^{*}, \dot{z}_{n}^{*}\right]^{T}$ )

$$
\begin{aligned}
\dot{e}_{i} & =e_{i+1}, \quad i \in\{0, \ldots, n-1\} \\
\dot{e}_{n} & =\beta\left(\boldsymbol{\Pi}, \boldsymbol{\Pi}_{o}, \overline{\mathbf{e}}, \underline{\mathbf{z}}^{*}\right)
\end{aligned}
$$

\footnotetext{
${ }^{11}$ The difference between $\mathbf{z}$ and $\zeta$ may not affect the whole state depending on $\boldsymbol{F}(\boldsymbol{\Pi}, \mathbf{x})$.
} 
and therefore as (defining $\underline{\mathbf{Z}}=\left[\mathbf{z}^{* T}, \dot{z}_{n}^{*}, \boldsymbol{\Pi}_{o}^{T}, \boldsymbol{\Pi}^{T}\right]^{T}$ )

$$
\dot{\overline{\mathbf{e}}}=\Upsilon(\overline{\mathbf{e}}, \underline{\mathbf{Z}})
$$

where $\underline{\mathbf{Z}}$ plays the role of an input to the augmented tracking error system in $\overline{\mathbf{e}}$. Since (33) is of nonlinear nature, known theory of robustness of linear systems can not be applied. However, the robust stability of the presented control law (28) can be analyzed by making use of a result which was primarily introduced by Kelemen [5], reinterpreted by Khalil and Kokotović [13] and elaborated by Lawrence and Rugh [14]. The version by Lawrence and Rugh [14] (to which the reader is referred for further details) is applied in the sequel.

Theorem 5.1: If the initial error $\overline{\mathbf{e}}(0)$, the velocity of the desired trajectory and the velocity of the exogenous parameters $\underline{\mathbf{Z}}$ are not too large, then the augmented tracking error $\overline{\mathbf{e}}$ is uniformly bounded. Moreover, if the desired trajectory of the flat output reaches a given point $z^{*}(t)=z_{\infty}^{*}, \forall t \geqslant t^{*}>0$ and the exogenous perturbations are constant from this point onwards $\left(\varpi_{o}(t)=\varpi_{o \infty}, \varpi(t)=\varpi_{\infty}, \forall t \geqslant t^{*}>0\right.$, then the tracking error e converges exponentially to zero.

Proof: In order to apply the stability results of [14] based on Lyapunov stability theory, their hypotheses (H1)-(H3) have to be verified:

- (H1): In (33), $\Upsilon$ is by construction at least $\mathcal{C}^{2}$.

- (H2): Define $\Gamma \subset \mathbb{R}^{n+1+2 q n}$ to be any bounded, open and connected subset of $\mathbb{R}^{n+1+2 q n}$ such that $\forall t \in[0, \infty), \underline{\mathbf{Z}} \in \Gamma$. Define also the continuously differentiable function $\boldsymbol{\xi}: \bar{\Gamma} \rightarrow \mathbb{R}^{n+1}$ such that $\Upsilon(\boldsymbol{\xi}(\boldsymbol{\zeta}), \boldsymbol{\zeta})=0$, for all $\boldsymbol{\zeta} \in \Gamma$ and $\Upsilon$ as defined in (33). The smoothness of $\underline{\mathbf{Z}}$ ensures the existence of $\Gamma$ and $\boldsymbol{\xi}$. The map $\boldsymbol{\xi}$ is nothing but a parametrization of equilibria of (33) corresponding to frozen-time inputs $\zeta \in \Gamma$. In view of the particular structure of (33) displayed in (32) one obviously has

$$
\boldsymbol{\xi}(\boldsymbol{\zeta})=\left[\xi_{0}(\boldsymbol{\zeta}), 0, \ldots, 0\right]^{T}, \forall \boldsymbol{\zeta} \in \Gamma
$$

where $\xi_{0}$ takes values in $\mathbb{R}$. If the interval containing all solutions $\xi_{0}(\boldsymbol{\zeta})$ is needed for the calculations presented in (H3), the implicit equation $\beta\left(\boldsymbol{\Pi}, \boldsymbol{\Pi}_{o}, \boldsymbol{\xi}(\boldsymbol{\zeta}), \boldsymbol{\zeta}\right)=0$ has to be solved by interval analysis. ${ }^{12}$

- (H3): To verify the eigenvalues of $\frac{\partial \Upsilon}{\partial \overline{\mathbf{e}}}$ around the parametrization of equilibria $\boldsymbol{\xi}(\boldsymbol{\zeta})$ of (34) and thus for all $\zeta \in \Gamma$, consider (31) to get

$$
\left.\left.\frac{\partial \Upsilon}{\partial \overline{\mathbf{e}}}\right|_{\Gamma} \doteq \frac{\partial \Upsilon}{\partial \overline{\mathbf{e}}}\right|_{\boldsymbol{\xi}(\boldsymbol{\zeta})}=\left[\begin{array}{ccccc}
0 & 1 & 0 & \cdots & 0 \\
0 & 0 & 1 & \cdots & 0 \\
\vdots & & & \ddots & \vdots \\
0 & 0 & 0 & \cdots & 1 \\
\mu_{0} & \mu_{1} & \mu_{2} & \cdots & \mu_{n}
\end{array}\right]
$$

where $\mu_{i}=\mu_{i}(\boldsymbol{\zeta})=\left.\frac{\partial \alpha}{\partial e_{i}}\right|_{\Gamma}, \boldsymbol{\zeta} \in \Gamma$, and $\alpha$ as given in (31). Thus the following characteristic polynomial containing the interval parameters $\tilde{\boldsymbol{\Pi}}$ and $\tilde{\boldsymbol{\Pi}}_{o}$ determines the possible root loci of the eigenvalues of $\left.\frac{\partial \Upsilon}{\partial \overline{\mathbf{e}}}\right|_{\Gamma}$ :

$$
\mathcal{P}\left(\boldsymbol{\Pi}_{o}, \boldsymbol{\Pi}\right)=s^{n+1}-\mu_{n} s^{n}-\cdots-\mu_{0}
$$

To determine whether the polynomial $\mathcal{P}(\varpi)$ of (36) has only zeros with real parts less than $-\lambda, \lambda>0$ (and thus whether (H3) is satisfied), one of the following methods is applied:

1. If the coefficients of the characteristic polynomial are algebraically independent with respect to the interval parameters, the stability can be deduced analytically in a necessary and sufficient way by the well-known theorem by Kharitonov [16].

2. In the case, in which the coefficients of the characteristic polynomial are algebraically dependent with respect to the interval parameters, the stability can be determined algebraically by the theorem of Frazer and Duncan [17] (it is remarked, that for $\Pi=\Pi_{o}$, there exists by construction at least one stable characteristic polynomial of (36), see Sec. IV).

\footnotetext{
${ }^{12}$ For a numerical solution, the authors propose to use the algorithm SIVIAX of [15, p. 104].
} 
3. Furthermore a numerical algorithm was developed by Walter and Jaulin [18], which is based on interval analysis. It can also be found in [15], where it is additionally combined with constraint propagation to shorten significantly the calculation time. Moreover, a new value set approach based on the knowledge of at least one stable characteristic polynomial is presented in [15].

As the hypotheses (H1)-(H3) of [14] are satisfied, there exists a $\rho>0$, a $T>0$, a $\delta_{1}(\rho)>0$ and a $\delta_{2}(\rho, T)>0$ such that

$$
\begin{aligned}
\|\overline{\mathbf{e}}(0)\| & <\delta_{1} \\
\frac{1}{T} \int_{t}^{t+T}\|\underline{\mathbf{Z}}(\tau)\| d \tau & <\delta_{2}, \quad t \geq 0
\end{aligned}
$$

then the corresponding solution $\overline{\mathbf{e}}$ of (33) satisfies

$$
\|\overline{\mathbf{e}}(t)\|<\rho, \quad t \geq 0
$$

that is the system (1) is stable under the tracking control law (28).

Applying the Corollary of [14] in the case $\underline{\mathbf{Z}}=\underline{\mathbf{Z}}_{\infty}, \forall t>t^{*}>0$ implies, in view of the structure of (34), that $\lim _{t \rightarrow \infty} \mathbf{e}(t)=0$ and $\overline{\mathbf{e}}\left(t^{*}\right)$ lies inside the domain of attraction ${ }^{13}$ of the exponentially stable equilibrium given by $\left[\xi_{0}(\boldsymbol{\zeta}), 0, \ldots, 0\right]^{T}$ evaluated at $\boldsymbol{\zeta}=\left[z_{\infty}^{*}, 0, \ldots, 0\right]^{T}$.

Remark 5.1: Note that the condition (38) assures, that robust stability can be proven if the velocity of exogenous perturbation and its derivatives $\dot{\Pi}$ (and their respective nominal counterparts) is not too big in an average sense. Since Heaviside jumps with microstructure are considered for the model of the exogenous perturbations, this might lead - depending on the system - to robustness in a lot of cases, even if derivatives of the perturbation $\Pi$ enter equation (9).

Remark 5.2: The first component $\xi_{0}(\zeta)$ of the parametrized manifold in (34) corresponds to the state of the integral of the feedback part of (28). Since $\xi_{i}(\zeta)=0, i=1, \ldots, n, \zeta \in \Gamma$, the other components of the manifold correspond to a zero tracking error $\mathbf{e}=\mathbf{0}$.

Remark 5.3: For the calculations within the mentioned methods, not only $\tilde{\boldsymbol{\Pi}}, \tilde{\boldsymbol{\Pi}}_{o}$ and $\xi_{0}$, but also $\mathbf{z}^{*}$ can be understood as of interval type. More precisely, since $\mathbf{z}^{*}$ is a function of time, for a given time interval $^{14} t \in \tilde{t}=\left[t_{0}, t_{1}\right]$, the only interval parameter which has to be added to $\tilde{\Pi}, \tilde{\Pi}_{o}$ and $\xi_{0}$ for the analysis is therefore $\tilde{t}$.

Remark 5.4: Partial state feedback is possible with the control strategy (28). Its stability can also be analyzed in the context of Theorem 5.1 by setting the respective control law coefficients $\lambda_{i}=0, i \in$ $\{k+2, \ldots, n\}$ (as in (17)). The minimal number of derivative actions $k$ necessary (but not implicitly sufficient) for stability can be determined from $\left.\frac{\partial \Upsilon}{\partial \overline{\mathbf{e}}}\right|_{\Gamma}$ using the necessary condition for negativity of the eigenvalues, that is $\mu_{i}, \quad i \in\{0, \ldots, n\}<0$ (see (36)).

Remark 5.5: In general, the choice of the nominal exogenous perturbation within the given uncertainty interval influences the robustness behavior. It is important to remark, that this choice does not only affect the coefficients of the respective characteristic polynomial, but also the magnitude of the so-called "quasi-exogenous" perturbations, that stem from the desired trajectory injection. The magnitude of these perturbations evidently influences the convergence behavior, therefore the choice of the nominal value within the uncertainty interval has to be undertaken carefully.

\section{ACADEMIC EXAMPLES}

In this section, two academic examples are presented in order to highlight the different aspects of how the exogenous perturbation enters the respective equations (for non-academic examples of exact feedfoward application to real systems under exogenous perturbations, cf. the application to the induction machine [19], [20] and the application to industrial semi-batch reactors [21]). The first academic example concerns a stable system, whereas the second academic example treats an unstable system.

\footnotetext{
${ }^{13}$ This result is comparable to linear systems when using an integral part in a PI-controller: for constant perturbations the control law leads to exponential stability, for non-constant perturbations the control law leads to bounded stability.

${ }^{14}$ Consider for example a set point change in $\left[t_{0}, t_{1}\right]$ using spline functions.
} 


\section{A. Application to a stable system}

Given the single-input system

$$
\begin{aligned}
& \dot{x}_{1}=x_{2}+\varpi \\
& \dot{x}_{2}=-x_{2}^{3}+u
\end{aligned}
$$

with $\mathbf{x}(0)=[0,0]^{T}$. This control system is flat with $z=x_{1}$, and Eqns. (4) and (5) read as

$$
\begin{aligned}
& \mathbf{x}=[z, \dot{z}-\varpi]^{T} \\
& u=\ddot{z}+(\dot{z}-\varpi)^{3}-\dot{\varpi}
\end{aligned}
$$

Thus, for every given admissible nominal trajectory of the flat output $t \mapsto z^{*}(t)$, the family of nominal feedforwards is given by

$$
u^{*}=\ddot{z}^{*}+\left(\dot{z}^{*}-\varpi_{o}\right)^{3}-\dot{\varpi}_{o}=\ddot{z}^{*}+\left(\dot{z}^{*}\right)^{3}
$$

The corresponding state transformation (8)

$$
\mathbf{z}=\left[x_{1}, x_{2}+\varpi\right]^{T}
$$

leads to the control normal form (9)

$$
\begin{aligned}
& \dot{z}_{1}=z_{2} \\
& \dot{z}_{2}=-\left(z_{2}-\varpi\right)^{3}+u+\dot{\varpi}
\end{aligned}
$$

with the initial condition given by $\mathbf{z}_{0}=\left[x_{10}, x_{20}+\varpi(0)\right]^{T}$. As shown in the proof of Theorem 2.1 of Section II, the nominal input (43) linearizes the system if the nominal perturbation condition $\varpi=\varpi_{o}$ holds, and if the initial condition is consistent following Definition 2.1, i.e. $\mathbf{x}_{0}=\mathbf{z}_{0}^{*}$. This feedforward linearization implies $\mathbf{z}(t)=\mathbf{z}^{*}(t)$, and thus $\mathbf{x}(t)=\mathbf{x}^{*}(t)$.

In order to counteract the eventual exogenous perturbations, a control law is designed following Section III, in which first the assumption is made that the exogenous perturbations coincide with the nominal ones in system (40), that is $\varpi=\varpi_{o}$. In order to simplify notations, it is assumed in the ongoing, that no a priori information is known on the perturbation $\varpi \in \mathbb{R}$, thus the nominal values are

$$
\forall t: \varpi_{o}^{(j)}(t)=0, \quad j=\{0,1\}
$$

Then control law (18) reads as

$$
u=\ddot{z}^{*}+\sum_{i=0}^{2} \lambda_{i} e_{i}+\left(\dot{z}^{*}\right)^{3}
$$

with the tracking error definitions for $e_{i}$ as in (16).

Considering now Section IV, the assumption $\varpi=\varpi_{o}$ has to be relaxed and $\varpi \neq \varpi_{o}$ has to be studied. This leads to the approximate state (26)

$$
\zeta=\mathrm{x}
$$

and the approximate tracking error definition $\tilde{e}_{i}$ of (27). The control law (28) reads then as

$$
u=\ddot{z}^{*}+\sum_{i=0}^{2} \lambda_{i} \tilde{e}_{i}+\left(\dot{z}^{*}\right)^{3}
$$

To study the robustness of the system (40) under the control law (49) in the vicinity of the desired trajectory, the control law (49) is substituted into (45), which yields

$$
\begin{aligned}
& \dot{z}_{1}=z_{2} \\
& \dot{z}_{2}=-\left(z_{2}-\varpi\right)^{3}+\ddot{z}^{*}+\sum_{i=0}^{2} \lambda_{i} \tilde{e}_{i}+\left(z_{2}^{*}\right)^{3}+\dot{\varpi}
\end{aligned}
$$


with the initial condition given by $\mathbf{z}_{0}=\left[x_{10}, x_{20}+\varpi(0)\right]^{T}$. To find the real tracking error equation in $\mathbf{e}=\mathbf{z}-\mathbf{z}^{*}$, the relation (30) between $\tilde{\mathbf{e}}$ and e can be established as

$$
\begin{aligned}
\tilde{e}_{i} & =e_{i}, \quad i \in\{0,1\} \\
\tilde{e}_{2} & =e_{2}-\varpi,
\end{aligned}
$$

Now, the augmented tracking error system in $\overline{\mathrm{e}}$ can be denoted by

$$
\begin{aligned}
\dot{e}_{i} & =e_{i+1}, \quad i \in\{0,1\} \\
\dot{e}_{2} & =-\left(e_{2}+z_{2}^{*}-\varpi\right)^{3}+\sum_{i=0}^{1} \lambda_{i} \tilde{e}_{i}+\lambda_{2}\left(e_{2}-\varpi\right)+\left(z_{2}^{*}\right)^{3}+\dot{\varpi}
\end{aligned}
$$

Following Section V, the robustness analysis of the augmented tracking error system (53) can be led by applying to it the results of Lawrence and Rugh [14] based on Lyapunov stability theory. Their hypotheses (H1)-(H3) have to be verified:

- (H1): The vector field of $(53)$ is at least $\mathcal{C}^{2}$.

- (H2): The parametrization of equilibria (34) of (53) corresponding to frozen-time inputs $z_{2}^{*}, \varpi$ and $\dot{\varpi}$ is

$$
\boldsymbol{\xi}=\left[\frac{1}{\lambda_{0}}\left(\left(z_{2}^{*}-\varpi\right)^{3}+\lambda_{2} \varpi-\dot{\varpi}\right), 0,0\right]^{T}
$$

- (H3): To verify the eigenvalues of the linearization of (53) around the parametrization of equilibria (54), this linearization is calculated as

$$
\left.\left.\frac{\partial \Upsilon}{\partial \overline{\mathbf{e}}}\right|_{\Gamma} \doteq \frac{\partial \Upsilon}{\partial \overline{\mathbf{e}}}\right|_{\boldsymbol{\xi}(\boldsymbol{\zeta})}=\left[\begin{array}{ccc}
0 & 1 & 0 \\
0 & 0 & 1 \\
\lambda_{0} & \lambda_{1} & -3\left(z_{2}^{*}-\varpi\right)^{2}+\lambda_{2}
\end{array}\right]
$$

Thus the following characteristic polynomial containing the interval parameters $z_{2}^{*}$ and $\varpi$ determines the possible root loci of the eigenvalues of $\left.\frac{\partial \Upsilon}{\partial \overline{\mathbf{e}}}\right|_{\Gamma}$ :

$$
\mathcal{P}\left(z_{2}^{*}, \varpi\right)=s^{3}+\left(3\left(z_{2}^{*}-\varpi\right)^{2}-\lambda_{2}\right) s^{2}-\lambda_{1} s^{1}-\lambda_{0}
$$

To decide whether this polynomial of (56) has only zeros with real parts less than $-\lambda, \lambda>0$, it is not necessary to apply interval algebra in this case. Since after a transformation of $q=s+\lambda$, the application of a Routh-Hurwitz table yields the following conditions

$$
\begin{aligned}
\lambda_{2} & <3\left(z_{2}^{*}-\varpi\right)^{2}-3 \lambda \\
\lambda_{1} \lambda_{0}-\lambda_{0} & >\lambda^{3}-\lambda^{2}\left(3\left(z_{2}^{*}-\varpi\right)^{2}-\lambda_{2}\right) \\
\left.\left.\left(3 \lambda^{2}-2 \lambda\left(z_{2}^{*}-\varpi\right)^{2}-\lambda_{2}\right)-\lambda_{1}\right)\left(z_{2}^{*}-\varpi\right)^{2}-\lambda_{2}-3 \lambda\right) & >\lambda_{1} \lambda_{0}-\lambda_{0}-\lambda^{3} \\
& +\lambda^{2}\left(3\left(z_{2}^{*}-\varpi\right)^{2}-\lambda_{2}\right)
\end{aligned}
$$

which can easily be checked for a given situation.

In the following the desired trajectory is chosen as a spline such as

$$
\begin{aligned}
0 \leqslant t \leqslant t_{\mathrm{i}}, & z^{*}(t)=z_{\mathrm{i}} \\
t_{\mathrm{i}} \leqslant t \leqslant t_{\mathrm{f}}, & z^{*}(t)=\sum_{k=0}^{5} \zeta_{k}\left(t-t_{i}\right)^{k}, \quad \zeta_{k} \in \mathbb{R} \\
t_{\mathrm{f}} \leqslant t \leqslant+\infty, & z^{*}(t)=z_{\mathrm{f}}
\end{aligned}
$$

with $t_{\mathrm{i}}=1 \mathrm{~s}, t_{\mathrm{f}}=4 \mathrm{~s}, z_{\mathrm{i}}=0.0$ and $z_{\mathrm{f}}=1$. The corresponding trajectories $z^{*}, \dot{z}^{*}$ and $\ddot{z}^{*}$ are depicted in Fig 1. The controller gains are furthermore chosen as $\lambda_{0}=-343, \lambda_{1}=-147$, and $\lambda_{2}=-21$, the perturbation is chosen as a spline such as

$$
\begin{aligned}
0 \leqslant t \leqslant t_{\mathrm{i}}^{\prime}, & \varpi(t)=\varpi_{\mathrm{i}} \\
t_{\mathrm{i}}^{\prime} \leqslant t \leqslant t_{\mathrm{f}}^{\prime}, & \varpi(t)=\sum_{k=0}^{3} v_{k}\left(t-t_{i}\right)^{k}, \quad v_{k} \in \mathbb{R} \\
t_{\mathrm{f}}^{\prime} \leqslant t \leqslant+\infty, & z^{*}(t)=\varpi_{\mathrm{f}}
\end{aligned}
$$


with $t_{\mathrm{i}}^{\prime}=2.45 \mathrm{~s}, t_{\mathrm{f}}^{\prime}=2.55 \mathrm{~s}, \varpi_{\mathrm{i}}=0.0$ and $\varpi_{\mathrm{f}}=1.0$. The corresponding trajectories $\varpi$ and $\dot{\varpi}$ are shown in Fig. 2. The results of the application of the control law (49) to the system (40) are given in Fig. 3 for an initial condition $\mathbf{x}(0)=\left(\begin{array}{lll}0.35 & 0.0\end{array}\right)^{T}$.

The largest real part of eigenvalues of $\left.\frac{\partial \Upsilon}{\partial \overline{\mathrm{e}}}\right|_{\Gamma}(55)$ during the transition are depicted in Fig. 4 and shows the stability margin required in (H3). Thus, a good tracking and robustness of the application of the control law (49) to the system (40) can be concluded.
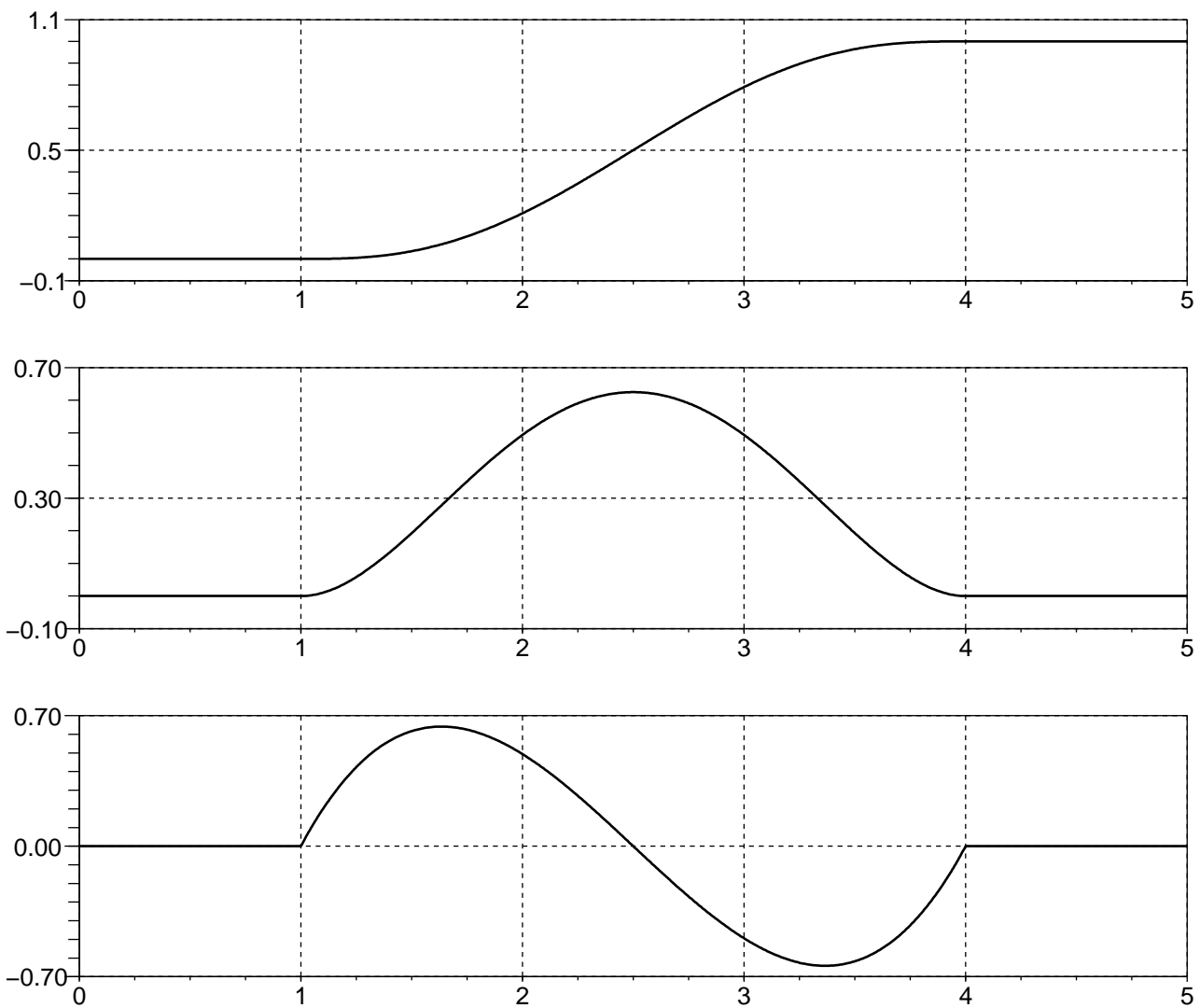

Fig. 1. Flat output predicted trajectory: $z^{*}, \dot{z}^{*}$ and $\ddot{z}^{*}$

\section{B. Application to an unstable system}

Consider now the unstable system (which is the unstable counterpart of the stable system (40))

$$
\begin{aligned}
& \dot{x}_{1}=x_{2}+\varpi \\
& \dot{x}_{2}=+x_{2}^{3}+u
\end{aligned}
$$

with $\mathbf{x}(0)=[0,0]^{T}$. Following the development in the preceding subsection, in this case the control law can be denoted as

$$
u=\ddot{z}^{*}+\sum_{i=0}^{2} \lambda_{i} e_{i}-\left(\dot{z}^{*}\right)^{3}
$$

The results of the application of the control law (61) to the system (60) is reported in Fig. 5, while the maximum real part of the eigenvalues associated with the closed-loop error equation is depicted in Fig. 6. One still remarks a control with good tracking and robustness properties. 

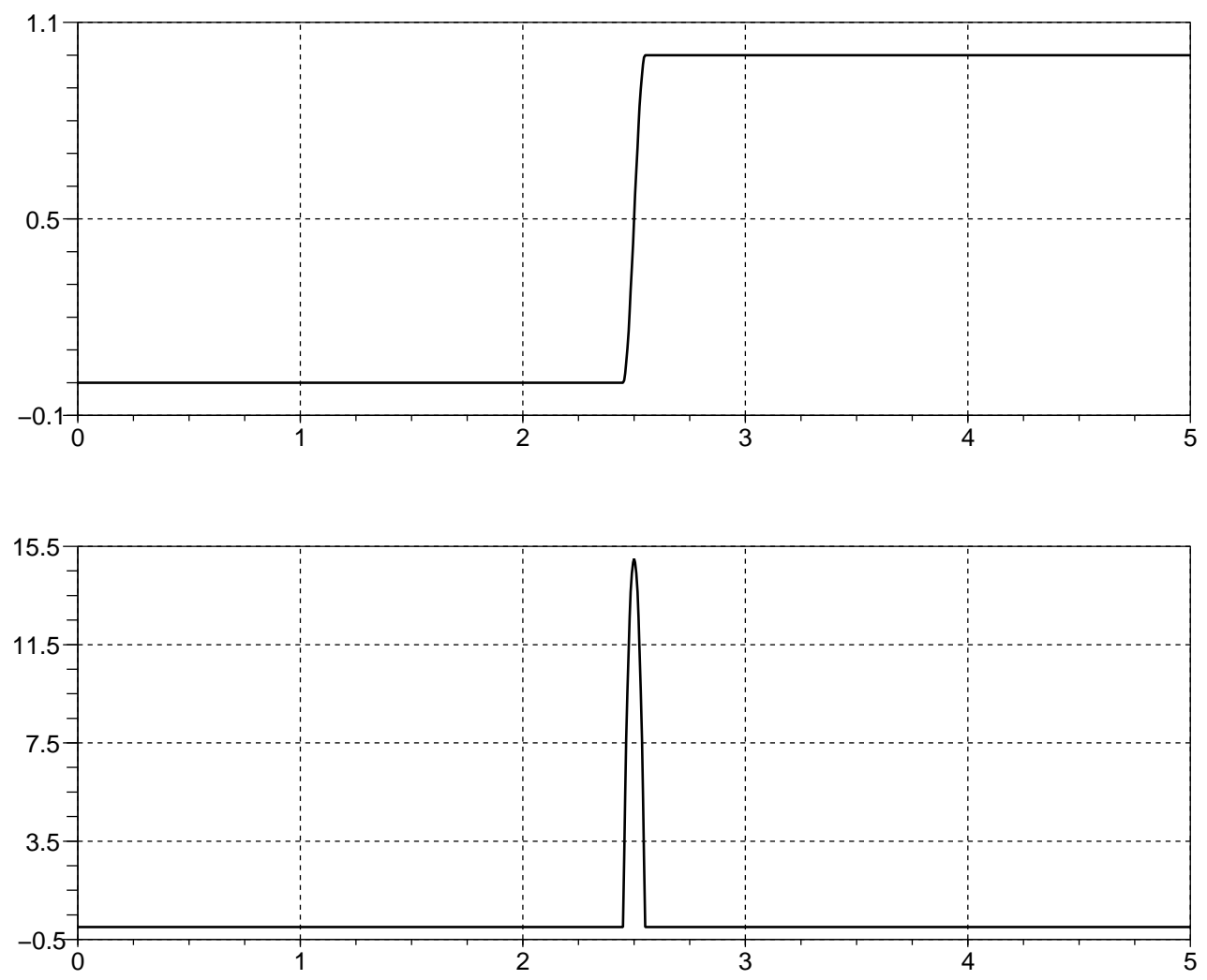

Fig. 2. Time trajectory of the perturbation: $\varpi$ and $\dot{\varpi}$

\section{CONCLUSION}

The article presents a method to analyze robust stability of exact feedforward linearization based on differential flatness with respect to exogenous perturbations and time-varying parameters. It is remarked, that representing the perturbed system in its so-called flat coordinates leads in all cases to the fulfillment of a sort of matching condition, that is the perturbation, its time derivatives and the input entering the system at the same point.

In case of using a PID-like stabilization for the feedback part of the combined control structure, the controller coefficients have to be traded-off with respect to the desired trajectory, its derivatives and generally the size of the uncertainty intervals of the exogenous perturbations or time-varying parameters. Also the choice of the nominal value of these parameters within the given uncertainty intervals is of interest for the performance of the closed loop system.

Modeling the exogenous perturbations by so-called "Heaviside jumps with microstructure" permits to stay close to physical reality. Since in the robustness result the velocity of the exogenous perturbation and its derivatives have to be bounded in an average sense, this might lead, depending on the system, to robustness in a lot of cases.

These results are important for the practicability in real applications of exact feedforward linearization based on differential flatness: ${ }^{15}$ in the case of a given flat nonlinear system, for which there already exists a linear PID-like controller stabilizing the system in the vicinity of an operation point, a nonlinear nominal feedforward based on flatness combined with the existing PID-like controller can lead to very good tracking of, for instance, guided set point changes even under exogenous perturbations.

\footnotetext{
${ }^{15}$ For successful applications of this method, cf. the application to the induction machine [19], [20] and the application to industrial semi-batch reactors [21].
} 


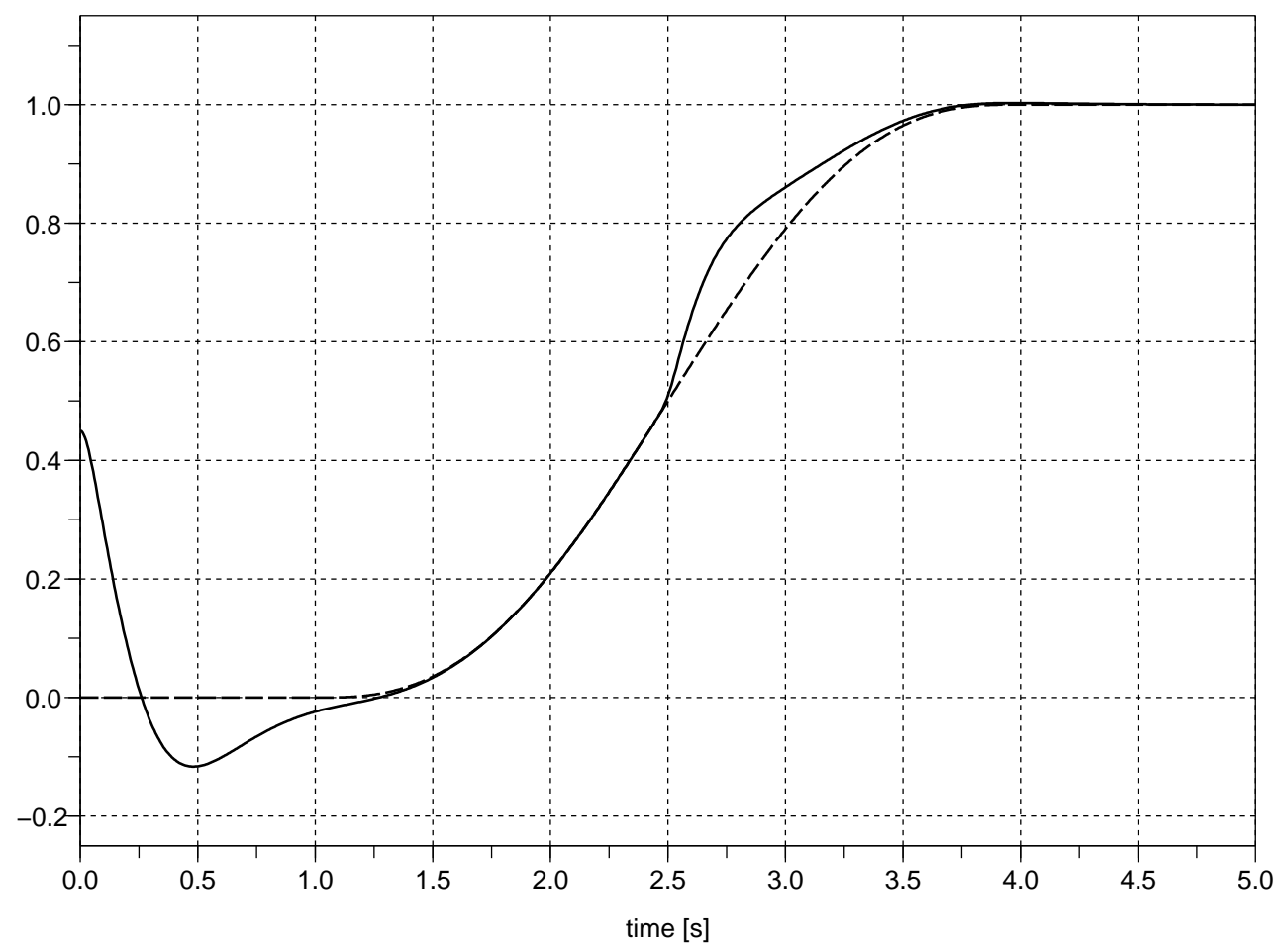

Fig. 3. Simulation result of the application of the control law (49) to the stable system (40): $x_{1}$ in solid line and $z^{*}$ in dashed line

\section{REFERENCES}

[1] H. Sira-Ramírez and S. K. Agrawal, Differentially Flat Systems. New York (NJ): Marcel Dekker, 2004.

[2] J. Lévine, Analysis and Control of Nonlinear Control Systems: A Flatness-based Approach. Berlin: Springer-Verlag, 2009.

[3] V. Hagenmeyer and E. Delaleau, "Exact feedforward linearization based on differential flatness," Internat. J. Control, vol. 76, pp. 537-556, 2003. [Online]. Available: http://www.informaworld.com/10.1080/0020717031000089570

[4] _ - "Robustness analysis of exact feedforward linearization based on differential flatness," Automatica, vol. 39, pp. 1941-1946, 2003.

[5] M. Kelemen, "A stability property," IEEE Trans. Autom. Control, vol. 31, pp. 766-768, 1986.

[6] J. Colombeau, Multiplication of Distributions. Berlin: Springer, 1992.

[7] M. Fliess, J. Lévine, P. Martin, and P. Rouchon, "Flatness and defect of non-linear systems: introductory theory and examples," Internat. J. Control, vol. 61, pp. 1327-1361, 1995.

[8] — _ "A Lie-Bäcklund approach to equivalence and flatness of nonlinear systems," IEEE Trans. Autom. Control, vol. 44, pp. 922-937, 1999.

[9] B. Jakubczyk and W. Respondek, "On linearization of control systems," Bull. Acad. Pol. Sci. Sér. Sci. Math., vol. 28, pp. 517-522, 1980.

[10] B. Charlet, J. Lévine, and R. Marino, “On dynamic feedback linearization,” Syst. Contr. Lett., vol. 13, pp. 143-151, 1989.

[11] H. K. Khalil, Nonlinear systems, 3rd ed. Upper Saddle River: Prentice-Hall, 1996.

[12] C. Reboulet and C. Champetier, "A new method for linearizing non-linear systems: the pseudolinearization," Internat. J. Control, vol. 40, pp. 631-638, 1984.

[13] H. K. Khalil and P. V. Kokotović, "On stability properties of nonlinear systems with slowly varying inputs," IEEE Trans. Autom. Control, vol. 36, pp. 229-229, 1991.

[14] A. L. Lawrence and W. J. Rugh, "On a stability theorem for nonlinear systems with slowly varying inputs," IEEE Trans. Autom. Control, vol. 35, pp. 860-864, 1990.

[15] L. Jaulin, M. Kieffer, O. Didrit, and E. Walter, Applied Interval Analysis. $\quad$ London: Springer, 2001.

[16] V. L. Kharitonov, "Asymptotic stability of an equilibrium position of a family of systems of linear differential equations," Differ. Uravn., vol. 14, pp. 2086-2088, 1978.

[17] R. Frazer and W. Duncan, "On the criteria for the stability of small motions," Proc. Roy. Soc. Edinburgh Sect. A, vol. 124, pp. 642-654, 1929.

[18] E. Walter and L. Jaulin, "Guaranteed characterization of stability domains via set inversion,” IEEE Trans. Autom. Control, vol. 39, pp. 886-889, 1994 


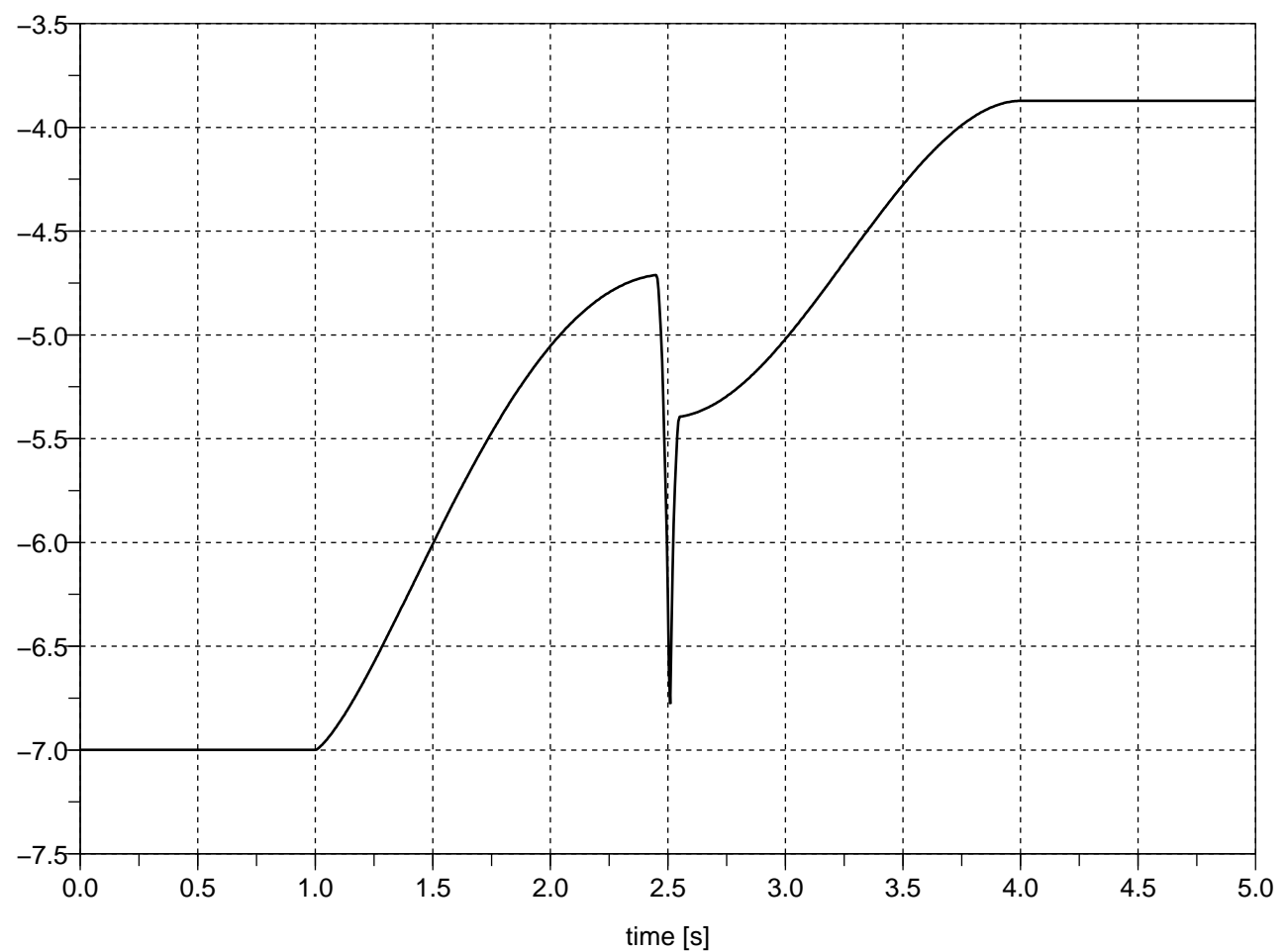

Fig. 4. Largest real part of eigenvalues of $\left.\frac{\partial \Upsilon}{\partial \overline{\mathbf{e}}}\right|_{\Gamma}$ (55) around the parametrization of equilibria (54)

[19] V. Hagenmeyer, A. Ranftl, and E. Delaleau, "Flatness-based control of the induction drive minimizing energy dissipation," in Nonlinear and Adaptive Control (NCN4 2001), A. Zinober and D. H. Owens, Eds. London: Springer, 2003, pp. 149-160.

[20] V. Hagenmeyer and E. Delaleau, "Continuous-time nonlinear flatness-based predictive control: an exact feedforward linearisation setting with an induction drive example," Internat. J. Control, vol. 81, pp. 1645-1663, 2008. [Online]. Available: http://www.informaworld.com/10.1080/00207170802090177

[21] V. Hagenmeyer and M. Nohr, "Flatness-based two-degree-of-freedom control of industrial semi-batch reactors using a new observation model for an Extended Kalman Filter approach,” Internat. J. Control, vol. 81, pp. 428-438, 2008. [Online]. Available: http://www.informaworld.com/10.1080/00207170701558951 


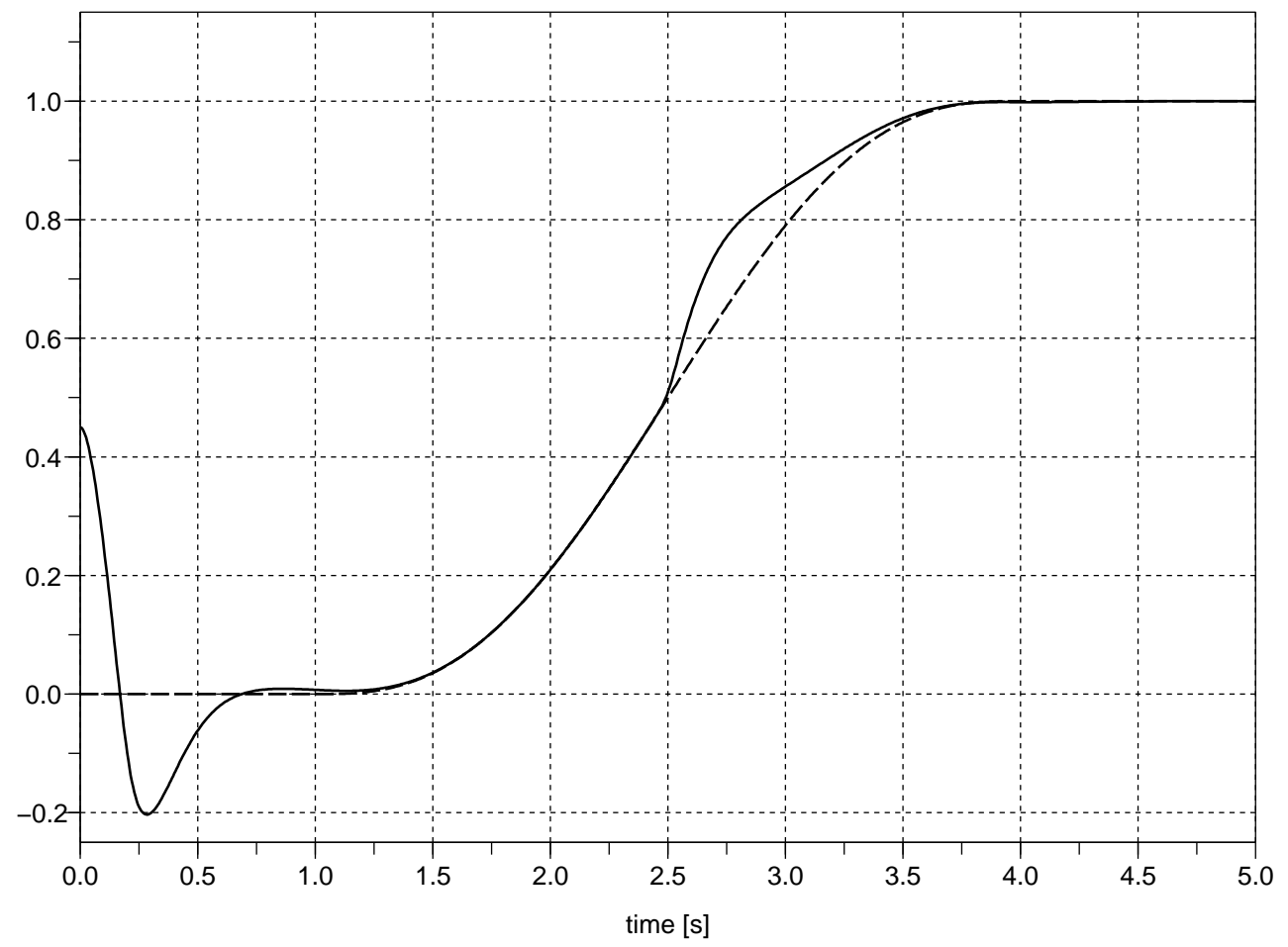

Fig. 5. Simulation result of the application of the control law (61) to the unstable system (60): $x_{1}$ in solid line and $z^{*}$ in dashed line 


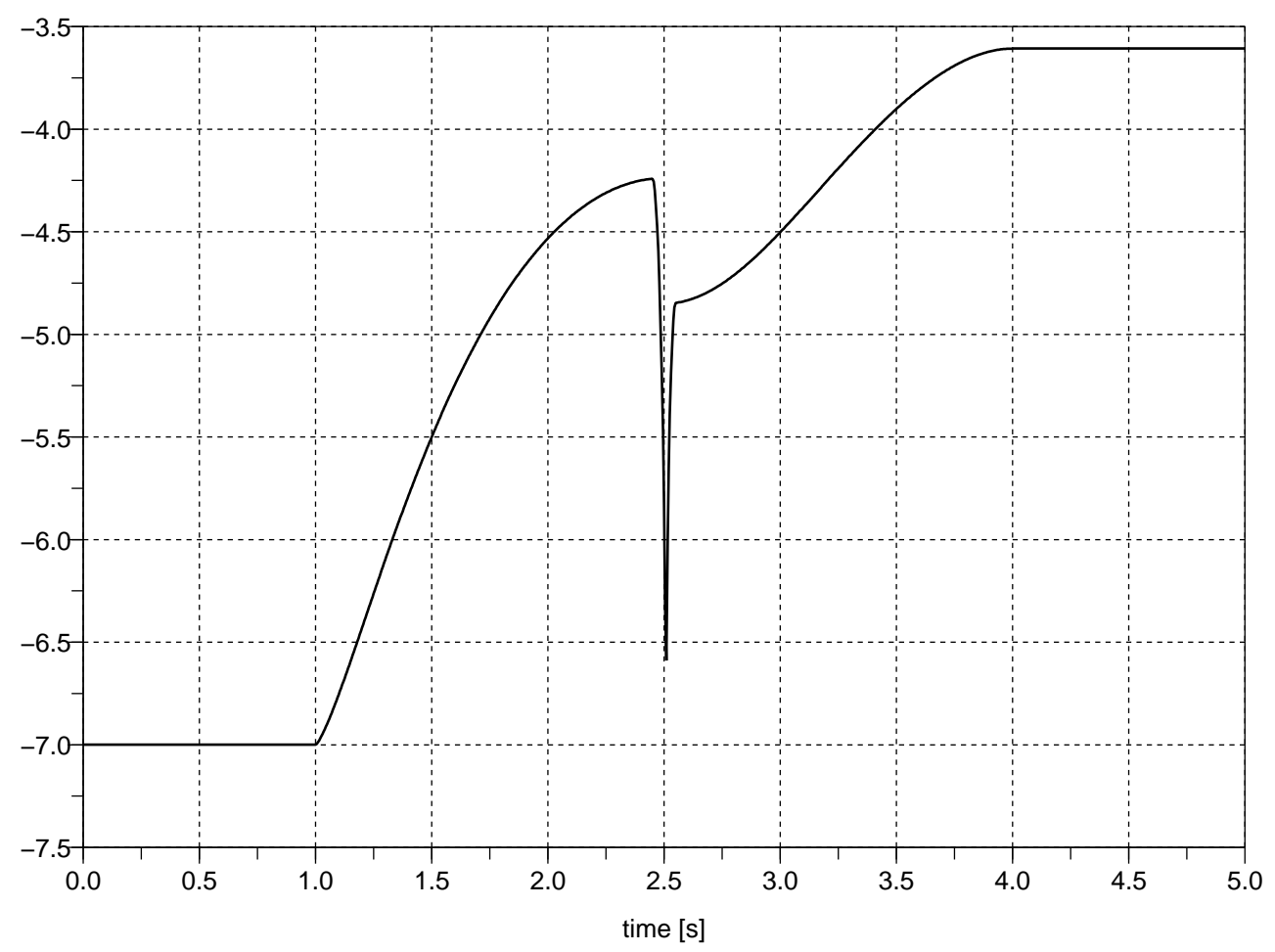

Fig. 6. Largest real part of eigenvalues of $\left.\frac{\partial \Upsilon}{\partial \overline{\mathbf{e}}}\right|_{\Gamma}$ around the respective parametrization of equilibria 